\title{
PREVALÊNCIA E FATORES ASSOCIADOS À DOENÇA DO REFLUXO GASTROESOFÁGICO
}

\author{
Sandro Schreiber de OLIVEIRA ${ }^{1}$, Iná da Silva dos SANTOS ${ }^{2}$, \\ José Francisco Pereira da SILVA ${ }^{3}$ e Eduardo Coelho MACHADO ${ }^{4}$
}

RESUMO - Racional - A doença de refluxo gastroesofágico é uma patologia de elevada prevalência, variando entre $21 \%$ e $56 \%$ em diferentes países. Objetivo - Estudar a epidemiologia e a prevalência da doença de refluxo gastroesofágico na população adulta (20 anos ou mais de idade) da cidade de Pelotas, RS. Metodologia - Estudo transversal de base populacional, com entrevistas domiciliares entre outubro de 1999 e janeiro de 2000. Analisou-se por regressão logística a associação da doença de refluxo gastroesofágico com idade, sexo, cor da pele, escolaridade, renda per capita, estado civil, variáveis emocionais (insônia, eventos estressantes negativos e mal-estar psicológico), índice de massa corporal, tabagismo e consumo semanal de bebidas alcoólicas. Resultados - Foram entrevistados 3.934 indivíduos, encontrando-se prevalência de 31,3\% (IC 95\% 29,9\% $-32,8 \%$ ). Após análise ajustada para controle de confusão encontrou-se associado com a doença de refluxo gastroesofágico o sexo feminino, viver sem companheiro(a), baixa escolaridade, presença de insônia, baixos índices de bem-estar psicológico, ocorrência de eventos estressantes e obesidade ou sobrepeso. Conclusão - A doença de refluxo gastroesofágico é uma patologia de elevada prevalência na população estudada, sendo o sexo, as variáveis antropométricas e as características emocionais, seus principais fatores associados.

DESCRITORES - Refluxo gastroesofágico, epidemiologia. Prevalência. Fatores de risco.

\section{INTRODUÇÃO}

A doença do refluxo gastroesofágico (DRGE), bem como os demais sintomas oriundos do sistema digestório superior são de alta freqüência na prática clínica diária. Ainda que suas complicações não sejam freqüentes, constituem-se, pela elevada prevalência, tema de relevância nas questões de saúde pública.

As taxas de prevalência de DRGE, caracterizada por presença de sintomas de pirose e regurgitação ácida, são bastante variáveis mas, principalmente em países ocidentais, quase sempre elevadas. Na Espanha, estudo transversal publicado em 2004, identificou prevalência de 31,6\% de DRGE ao ano ${ }^{(2)}$. Na Bélgica também é elevada, tendo sido de $28 \%$ em $2002^{(7)}$. Em estudo realizado na Austrália em 1996,56\% dos indivíduos relataram ter tido sintomas de DRGE pelo menos alguma vez em suas vidas, sendo que $37 \%$ os têm pelo menos uma vez a cada 4 meses $^{(1)}$. Na Dinamarca, em 1994, a prevalência foi de $38 \%$ entre os homens e $30 \%$ entre as mulheres ${ }^{(5)}$. No Brasil, em 2001 foi de 48,2\% ${ }^{(10)}$. Em estudo de revisão publicado em 1997, a variação da prevalência fica na faixa de $10 \%$ a $48 \%$ para pirose, $9 \%$ a $45 \%$ para regurgitação ácida e $21 \%$ a $59 \%$ para ambos os sintomas ${ }^{(4)}$.
Elevado número de consultas é originado a partir de sintomas de DRGE. Na Inglaterra, em 1994, 8\% das consultas médicas em atenção primária à saúde e $11 \%$ da demanda espontânea foram ocasionadas por sintomas do sistema digestório ${ }^{(3)}$. Em Autumn, na Alemanha, em estudo realizado no ano de 2000, $81,7 \%$ dos indivíduos com sintomas de DRGE já haviam realizado pelo menos uma gastroscopia para investigação de seus sintomas ${ }^{(14)}$. Estudo realizado entre trabalhadores na Itália, em 1999, mostrou que pelo menos $2,6 \%$ dos indivíduos sintomáticos têm seu trabalho afetado pelos sintomas ${ }^{(13)}$.

Estudos de base populacional com baixos índices de perdas ou recusas ainda são minoria entre as publicações sobre este tema. Os estudos para investigar fatores determinantes de DRGE são, em sua maioria, realizados em serviços de saúde, podendo indicar fatores agravantes e não necessariamente fatores de risco para os indivíduos da população que não procura ajuda médica para seus sintomas.

O presente estudo visou determinar a prevalência de sintomas de DRGE e contribuir para elucidação dos diferentes fatores de risco para essa patologia.

${ }^{1}$ Núcleo de Saúde Coletiva da Escola de Medicina da Universidade Católica de Pelotas; ${ }^{2}$ Departamento de Medicina Social da Faculdade de Medicina da Universidade Federal de Pelotas; ${ }^{3}$ Departamento de Clínica Médica da Universidade Federal de Pelotas; ${ }^{4}$ Hospital Escola da Universidade Federal de Pelotas, Pelotas, RS.

Endereço para correspondência: Dr. Sandro Schreiber de Oliveira - Rua Dom Pedro II, 663/502 - Centro - 96010-300 - Pelotas, RS. E-mail: episa@terra.com.br 


\section{METODOLOGIA}

Em 1999 e 2000, na cidade de Pelotas, RS, foi realizado um grande estudo transversal, com pelo menos 12 temas de interesse diferentes, em que foram entrevistados quase 6.000 indivíduos de todas as idades, através de processo amostral dos cerca de 250.000 habitantes residentes na zona urbana do município. A cidade de Pelotas localiza-se na metade sul do Rio Grande do Sul, sendo sua formação étnica baseada, principalmente, na imigração européia.

A população alvo desse estudo foram os adultos, com $20 \mathrm{ou}$ mais anos de idade, residentes na zona urbana.

Desejava-se entrevistar 3.717 indivíduos nesta faixa etária, amostra calculada a partir dos seguintes parâmetros: poder de $80 \%$ para detecção de um risco relativo de pelo menos 1,5 , significativo ao nível de $5 \%$, considerando-se pelo menos $10 \%$ de indivíduos expostos aos fatores em estudo e prevalência estimada de DRGE de 20\%. Foi acrescido, ainda, um fator de 1,5 para correção do efeito de delineamento amostral, 10\% para perdas e $30 \%$ para controle de variáveis de confusão.

Para que se encontrassem os 3.717 indivíduos necessários para o desenvolvimento do estudo, seria necessário visitar 2.100 domicílios, considerando a média de moradores adultos por domicílio, divulgada pelo Instituto Brasileiro de Geografia e Estatística (IBGE) a partir dos dados do censo demográfico de 1991. O processo amostral utilizado foi aleatório em estágios múltiplos.

Para escolha dos 2.100 domicílios a serem visitados, realizou-se uma amostragem sistemática, onde foram sorteados 48 dentre os 281 setores censitários da zona urbana do município de Pelotas. Em cada setor censitário foram visitados 44 domicílios e nestes entrevistavam-se todos os adultos residentes. A identificação dos domicílios a serem visitados foi feita a partir de visita e mapeamento de cada um dos setores censitários sorteados, ocasião em que todos os quarteirões foram numerados, bem como as esquinas de cada um destes. Aleatoriamente foram sorteados os quarteirões, e, nestes, a esquina pela qual se iniciaria o processo amostral de escolha dos domicílios. A partir da esquina sorteada, um entre os três primeiros domicílios era sorteado e, a partir deste, de maneira sistemática, eram visitados um de cada três domicílios.

O único critério de exclusão para o presente estudo foi a presença de doença física ou mental que causasse limitação na compreensão das perguntas ou respostas. Consideraram-se como perdas ou recusas, respectivamente, aqueles indivíduos que após, pelo menos, três visitas da entrevistadora em dias e horários diferentes e, no mínimo, uma do supervisor de campo, não puderam ser encontrados ou mantiveram a negação de resposta.

Para coleta dos dados, utilizou-se questionário padronizado, pré-testado e codificado, abordando além das variáveis necessárias à definição do desfecho, fatores socioeconômicos: renda per capita em salários mínimos da época, escolaridade em anos completos de aprovação na escola e estado civil (casado(a), solteiro(a), viúvo(a) ou separado(a)/divorciado(a)); fatores demográficos: sexo observado pelo entrevistador, cor da pele, classificada como branca ou nãobranca, e idade em anos completos; características emocionais: presença de insônia durante os últimos 30 dias, ocorrência de eventos estressantes durante o último ano (desemprego, separação, morte de familiar próximo, acidente pessoal grave, assalto ou roubo, presença de doença grave entre os moradores do domicílio) e bem- estar psicológico, medido por uma escala de sete faces ${ }^{(8)}$; variáveis comportamentais: freqüência semanal de uso de álcool, tabagismo, medido pelo número de cigarros fumados por dia, e índice de massa corporal (IMC), calculado através de peso e altura referidos.

Como definição de sintomas de DRGE utilizou-se o relato de ocorrência de pirose numa freqüência de, pelo menos, uma vez por semana durante o último ano. Essa definição tem sido considerada a mais sensível para DRGE. O relato de "amargor na boca" foi queixa associada e muito freqüente nos indivíduos deste estudo.

Vinte e duas entrevistadoras realizaram o trabalho de campo, que teve a duração de 4 meses. As entrevistadoras foram treinadas para aplicação do questionário em curso com duração de 40 horas.

Foi realizado controle de qualidade, através de re-entrevista de $10 \%$ dos indivíduos, pelo supervisor de campo, com objetivo de verificar a consistência de algumas informações e conferir o adequado cumprimento da logística do estudo. O resultado do teste de concordância entre as variáveis nas duas entrevistas (Kappa) foi considerado muito bom, na ordem de 0,79.

Utilizou-se o software SPSS/PC+ 8.0 para a análise por regressão logística, seguindo modelo teórico hierarquizado construído previamente e composto por três níveis: o primeiro, agregando as variáveis socioeconômicas e demográficas; o segundo, constituído pelas variáveis de perfil emocional; e o terceiro nível, com as variáveis comportamentais (tabagismo, consumo de álcool) e IMC. Inicialmente todas as variáveis entravam no modelo e, a cada nível hierárquico, eram retiradas aquelas que apresentavam valor $P$ maior que 0,20 . Desse modo, cada variável encontra-se ajustada para todas as do mesmo nível hierárquico no modelo e ainda para todas as outras dos níveis hierárquicos superiores.

Os dados foram também analisados considerando-se o efeito de delineamento. Em razão de ocorrer variação apenas na segunda casa decimal dos intervalos de confiança em relação à análise convencional, optou-se por apresentar os resultados sem a correção para o efeito do delineamento.

\section{RESULTADOS}

O processo amostral definido foi capaz de identificar 2.052 domicílios elegíveis para o estudo. Todos os domicílios foram visitados, tendo-se encontrado 4.203 indivíduos que preencheram os critérios de inclusão do estudo. Foi possível entrevistar 3.934 pessoas, perfazendo um índice de perdas e recusas de $6,8 \%$.

A Tabela 1 mostra que, entre os 3.934 indivíduos estudados, 2.243 (57\%) eram do sexo feminino. Sete por cento dos indivíduos possuíam 70 anos ou mais e a média de idade foi de cerca de 43 anos. Pouco mais de $80 \%$ dos indivíduos entrevistados referiram ser de cor branca.

Oito por cento dos entrevistados nunca freqüentaram a escola e a média de escolaridade da amostra foi de aproximadamente 7 anos e meio. A média de renda per capita chegava a quase três salários mínimos, porém $15 \%$ da amostra tinha renda per capita inferior a meio salário mínimo.

Insônia foi problema relatado por mais de um terço dos entrevistados e $60 \%$ haviam sofrido algum evento estressante nos últimos 12 meses. Ainda assim, mais de 50\% da amostra enquadraram-se nas faixas de bem-estar mais elevado, 
TABELA 1 - Distribuição de características da amostra e prevalência de sintomas de DRGE em Pelotas

\begin{tabular}{|c|c|c|}
\hline Característica & $\mathrm{n}$ & Sintomas de DRGE \\
\hline Sexo & & $<0,001$ \\
\hline Feminino & $2.243(57,0 \%)$ & 34,3 \\
\hline Masculino & $1.691(43,0 \%)$ & 27,4 \\
\hline Cor da Pele & & 0,05 \\
\hline Branca & $3.169(80,6 \%)$ & 30,6 \\
\hline Não-branca & $765(19,4 \%)$ & 34,2 \\
\hline Estado Civil & & $<0,001$ \\
\hline Casado & $2.498(63,5 \%)$ & 32,5 \\
\hline Solteiro & $795(20,2 \%)$ & 22,9 \\
\hline Viúvo & $322(8,2 \%)$ & 36,6 \\
\hline Separado/divorciado & $319(8,1 \%)$ & 38,2 \\
\hline Idade & & $<0,001 *$ \\
\hline 20-29 & $921(23,4)$ & 25,4 \\
\hline $30-39$ & $852(21,7 \%)$ & 30,4 \\
\hline $40-49$ & $870(22,1 \%)$ & 32,3 \\
\hline $50-59$ & $598(15,2 \%)$ & 35,5 \\
\hline $60-69$ & $415(10,5 \%)$ & 38,8 \\
\hline Mais de 69 & $278(7,1 \%)$ & 30,9 \\
\hline Anos de escolaridade & & $<0,001 *$ \\
\hline Não foi a escola & $327(8,3 \%)$ & 46,8 \\
\hline 1 a 4 & $846(21,5 \%)$ & 37,8 \\
\hline 5 a 8 & $1.281(32,6 \%)$ & 33,6 \\
\hline 9 a 11 & $795(20,2 \%)$ & 26 \\
\hline Mais de 11 & $682(17,3 \%)$ & 18 \\
\hline Renda per capita & & $<0,001 *$ \\
\hline 0 a 0,5 & $575(14,6 \%)$ & 40,2 \\
\hline 0,6 a 1 & $873(22,2 \%)$ & 35,5 \\
\hline 1,1 a 2 & $1.007(25,6 \%)$ & 31,1 \\
\hline 2,1 a 3 & $462(11,7 \%)$ & 27,9 \\
\hline 3,1 a 5 & $418(10,6 \%)$ & 24,9 \\
\hline Mais de 5 & $532(13,5 \%)$ & 24,4 \\
\hline Insônia & & $<0,001$ \\
\hline Não & $2.546(64,7 \%)$ & 23,6 \\
\hline Sim & $1.388(35,3 \%)$ & 45,5 \\
\hline Bem-estar & & $<0,001 *$ \\
\hline Alto & $2.036(51,8 \%)$ & 25,3 \\
\hline Intermediário & $1.531(38,9 \%)$ & 34,5 \\
\hline Baixo & $318(8,1 \%)$ & 53,8 \\
\hline Eventos estressantes & & $<0,001 *$ \\
\hline Nenhum & $1.558(39,6 \%)$ & 28,3 \\
\hline $\mathrm{Um}$ & $1.530(38,9 \%)$ & 30,5 \\
\hline Dois & $630(16,0 \%)$ & 37,8 \\
\hline Três ou mais & $209(5,3 \%)$ & 41,1 \\
\hline Freqüência de uso de álcool & & $0,001 *$ \\
\hline Não usa & $1.902(48,3 \%)$ & 35,1 \\
\hline Menos de 3 vezes/semana & $1.582(40,2 \%)$ & 26,9 \\
\hline $3-6$ vezes & $237(6,0 \%)$ & 32,1 \\
\hline Diário & $208(5,3 \%)$ & 29,3 \\
\hline IMC & & $<0,001 *$ \\
\hline$<20$ & $277(7,0 \%)$ & 28,2 \\
\hline $20-24,99$ & $1.570(39,9 \%)$ & 27,9 \\
\hline $25-29,99$ & $1.223(31,1 \%)$ & 32,1 \\
\hline 30 ou mais & $580(14,7 \%)$ & 39,1 \\
\hline Fumo & & $0,02 *$ \\
\hline Não-fumante & $2.805(71,3 \%)$ & 30,4 \\
\hline Até 5 & $219(5,6 \%)$ & 34,7 \\
\hline 6 a 10 & $269(6,8 \%)$ & 30,1 \\
\hline 11 a 15 & $132(3,4 \%)$ & 25,8 \\
\hline 16 a 20 & $346(8,8 \%)$ & 35,8 \\
\hline Mais de 20 & $155(3,9 \%)$ & 39,4 \\
\hline
\end{tabular}

* Valor $P$ de tendência linear quando perguntado sobre seu estado durante a maior parte do último ano.

Mais de $25 \%$ das pessoas elegíveis eram fumantes e quase metade delas referiu ingestão regular semanal de bebidas alcoólicas no último mês. Cerca de $45 \%$ dos indivíduos encontravam-se numa faixa de IMC adequada e 15,6\% apresentavam-se obesos, de acordo com a classificação do IMC.

A prevalência de DRGE no último ano foi de $31,3 \%$ (IC 95\% 29,9-32,8).

Observando ainda a Tabela 1, pode-se verificar a prevalência dos sintomas de DRGE e sua distribuição para cada uma das variáveis independentes. Os sintomas de DRGE são mais freqüentes entre os não-brancos que apresentam maior prevalência $(34,2 \%)$, enquanto os indivíduos de cor branca têm $30,6 \%$, com valor $P$ limítrofe de 0,05 . Quanto ao sexo, a prevalência foi bem mais elevada entre as mulheres $(34,3 \%)$ do que entre os homens $(27,4 \%)$. Com relação ao estado civil, os indivíduos solteiros $(22,9 \%)$ ou casados $(32,5 \%)$ apresentaram menor prevalência do que os viúvos $(36,6 \%)$ e os separados/divorciados $(38,2 \%)$. Na análise da idade dos entrevistados, verificou-se uma relação direta de aumento das taxas de prevalência conforme aumentava a faixa etária dos indivíduos ( $P$ de tendência linear $<0,001$ ). Porém, para os indivíduos acima de 69 anos, observou-se redução importante (30,9\%), voltando a apresentar taxa semelhante a do grupo com 30 a 39 anos. Para a escolaridade e a renda per capita houve diminuição da prevalência conforme aumentava a renda e a escolaridade, apresentando $P$ de tendência linear em ambos os casos $<0,001$.

De maneira geral, taxas mais elevadas de prevalência de sintomas de DRGE encontram-se significativamente associadas com o sexo feminino, cor não-branca, estado civil (viúvo ou separado/divorciado), menor escolaridade e renda, faixas etárias mais elevadas, insônia no último mês, baixos índices de bem-estar psicológico, ocorrência de eventos estressantes no último ano, IMC mais elevado, tabagismo e consumo de bebidas alcoólicas.

Entre os indivíduos que apresentavam insônia, encontra-se a maior diferença entre as taxas de prevalência de sintomas de DRGE, 45,5\% entre os insones e 23,6\% entre os demais, com valor $P$ altamente significativo $<0,001$. A ocorrência de sintomas de DRGE entre os que sofreram eventos estressantes no último ano, obedece a uma relação linear de aumento da prevalência conforme aumentava o número de eventos estressantes ocorridos, com $P$ de tendência linear menor que 0,001 . Situação semelhante ocorre quando se analisa escala de bem-estar psicológico. Os indivíduos com melhores índices de bem-estar apresentavam prevalência de $25,3 \%$ de sintomas de DRGE, enquanto que os com piores índices tinham um coeficiente da ordem de 53,8\%, igualmente com importante associação estatística de tendência linear.

A associação com IMC obedeceu tendência linear com significância estatística $(P<0,001)$, onde os indivíduos com IMC acima de 30 apresentavam prevalência de $39,1 \%$, comparada com $28,2 \%$ entre os com IMC menor de 20 .

A prevalência de sintomas de DRGE encontrou-se igualmente associada linearmente ao hábito de fumar. Indivíduos que fumavam mais cigarros por dia apresentavam maiores taxas de prevalência, sendo de quase 10 pontos percentuais a diferença entre fumantes de mais de 20 cigarros ao dia e os não-fumantes. 
Quanto ao consumo de bebidas alcoólicas, encontrou-se associação estatisticamente significativa. Os indivíduos que relatavam ingestão menos freqüente de bebida alcoólica (menos de 3 vezes por semana), tiveram menor prevalência que os demais bebedores (26,9\%), versus $32,1 \%$ e $29,3 \%$, respectivamente, entre os que consumiam de 3 a 6 vezes por semana e diariamente, enquanto que os que não consumiam álcool tiveram prevalência de $35,1 \%$.

Na Tabela 2 observam-se os efeitos brutos das variáveis independentes sobre a prevalência de sintomas de DRGE. Dentre as variáveis dicotômicas, o sexo feminino está associado a aumento de $39 \%$ do risco de apresentar sintomas; a cor não-branca, a um

TABELA 2 - Sintomas de DRGE conforme os fatores de risco estudados em Pelotas, análise bruta

\begin{tabular}{|c|c|c|c|}
\hline Característica & Razão de chance & Intervalo de confiança 95\% & $P$ \\
\hline \multicolumn{4}{|l|}{ Sexo } \\
\hline Feminino & 1,39 & $1,20-1,59$ & $<0,001$ \\
\hline Masculino & 1 & & \\
\hline \multicolumn{4}{|l|}{ Cor da pele } \\
\hline Branca & 1 & & 0,05 \\
\hline Não-branca & 1,18 & $1,00-1,39$ & \\
\hline \multicolumn{4}{|l|}{ Idade } \\
\hline $20-29$ & 0,76 & $0,57-1,02$ & \\
\hline $30-39$ & 0,98 & $0,73-1,30$ & \\
\hline $40-49$ & 1,07 & $0,80-1,43$ & $<0001$ \\
\hline $50-59$ & 1,23 & $0,90-1,66$ & \\
\hline $60-69$ & 1,42 & $1,02-1,95$ & \\
\hline Mais de 69 & 1 & & \\
\hline \multicolumn{4}{|l|}{ Estado civil } \\
\hline Casado & 1 & & \\
\hline Solteiro & 0,62 & $0,51-1,53$ & 0,001 \\
\hline Viúvo & 1,20 & $0,94-1,53$ & \\
\hline Separado/divorciado & 1,29 & $1,01-1,64$ & \\
\hline \multicolumn{4}{|l|}{ Anos de escolaridade } \\
\hline Não foi a escola & 4 & $2,98-5,35$ & \\
\hline 1 a 4 & 2,76 & $2,17-3,51$ & $<0,001$ \\
\hline 5 a 8 & 2,30 & $1,82-2,88$ & \\
\hline 9 a 11 & 1,60 & $1,24-2,06$ & \\
\hline Mais de 11 & 1 & & \\
\hline \multicolumn{4}{|l|}{ Renda per capita } \\
\hline 0 a 0,5 & 2,08 & $1,60-2,69$ & \\
\hline 0,6 a 1 & 1,70 & $1,34-2,17$ & \\
\hline 1,1 a 2 & 1,39 & $1,10-1,77$ & $<0,001$ \\
\hline 2,1 a 3 & 1,20 & $0,90-1,59$ & \\
\hline 3,1 a 5 & 1,02 & $0,76-1,38$ & \\
\hline Mais de 5 & 1 & & \\
\hline \multicolumn{4}{|l|}{ Insônia } \\
\hline Não & 1 & & $<0,001$ \\
\hline Sim & 2,71 & $2,35-3,11$ & \\
\hline \multicolumn{4}{|l|}{ Bem-estar } \\
\hline Alto & 1 & & $<0,001$ \\
\hline Intermediário & 1,55 & $1,34-1,79$ & \\
\hline Baixo & 3,42 & $2,69-4,36$ & \\
\hline Eventos estressantes & & & $<0,001$ \\
\hline Nenhum & 1 & & \\
\hline Um & 1,11 & $0,95-1,30$ & \\
\hline Dois & 1,54 & $1,27-1,87$ & \\
\hline Três ou mais & 1,77 & $1,32-2,38$ & \\
\hline \multicolumn{4}{|l|}{ IMC } \\
\hline$<20$ & 1 & & \\
\hline $20-24,99$ & 0,99 & $0,74-1,31$ & $<0,001$ \\
\hline $25-29,99$ & 1,21 & $0,91-1,61$ & \\
\hline 30 ou mais & 1,64 & $1,21-2,24$ & \\
\hline \multicolumn{4}{|l|}{ Fumo } \\
\hline Não-fumante & 1 & & \\
\hline até 5 & 1,22 & $0,91-1,62$ & \\
\hline 6 a 10 & 0,99 & $0,75-1,30$ & 0,03 \\
\hline 11 a 15 & 0,79 & $0,53-1,18$ & \\
\hline 16 a 20 & 1,28 & $1,01-1,62$ & \\
\hline Mais de 20 & 1,49 & $1,07-2,07$ & \\
\hline \multicolumn{4}{|c|}{ Freqüência de uso de álcool } \\
\hline Não usa & 1,31 & $0,95-1,78$ & \\
\hline $\begin{array}{l}\text { Menos de } 3 \text { vezes/ } \\
\text { semana }\end{array}$ & 0,89 & $0,64-1,22$ & $<0,001$ \\
\hline $3-6$ vezes & 1,14 & $0,76-1,70$ & \\
\hline Diário & 1 & & \\
\hline
\end{tabular}

aumento de $18 \%$, e a ocorrência de insônia aumenta em 2,71 vezes o risco do indivíduo apresentar DRGE.

O risco bruto cresce linearmente com a idade, o IMC, a intensidade do hábito de fumar, a freqüência de consumo de bebidas alcoólicas e o número de eventos estressantes vivenciados no último ano.

Entre os indivíduos com 60 a 69 anos de idade, o risco é $42 \%$ maior do que o verificado entre os maiores de 69 anos, tomados como referência. Entre os com menos de 40 anos de idade, o risco é menor do que entre os demais grupos etários.

O risco aumenta com o IMC, sendo $21 \%$ e $64 \%$ maior entre indivíduos com IMC, respectivamente, de 25,00 a 29,99, e de maior ou igual a 30, comparativamente àqueles com IMC menor que 20.

Os fumantes de mais de 20 cigarros por dia têm risco $49 \%$ maior de serem sintomáticos do que os não-fumantes. Indivíduos que fumaram no máximo 5 cigarros por dia, no entanto, apresentam risco maior do que o verificado entre os fumantes de 6 a 10 cigarros por dia e 11 a 15 cigarros por dia (respectivamente, razão de chance $=1,22 ; 0,99$ e 0,79 ).

$\mathrm{O}$ risco bruto de DRGE entre não-consumidores de bebidas alcoólicas foi $31 \%$ maior do que o encontrado entre os bebedores diários. A freqüência de consumo menor do que 3 vezes por semana apresentou efeito bruto protetor, com risco $11 \%$ menor do que o observado entre bebedores diários.

O risco de DRGE foi crescente com o número de eventos estressantes experimentados no último ano. Aqueles com história de três ou mais eventos apresentaram risco $77 \%$ maior do que o observado entre os controles (indivíduos com nenhum evento).

A renda per capita e a escolaridade apresentaram associação inversa à presença de sintomas de DRGE. Entre os analfabetos, o risco foi 4 vezes maior do que entre indivíduos com 11 anos ou mais de escolaridade. O primeiro grau completo e incompleto associaramse a risco 2 vezes maior de DRGE do que os controles.

$\mathrm{O}$ risco associado à renda inferior a meio salário mínimo foi 2 vezes maior do que o encontrado para indivíduos com renda mensal superior a cinco salários mínimos.

Entre os viúvos e os separados/divorciados, o risco foi de $20 \%$ a $30 \%$ maior do que entre os casados. E os que apresentaram baixo escore de bem-estar psicológico, tiveram risco 3,42 vezes maior do que o observado entre aqueles com escore alto.

Quando se controlam os fatores de confusão (Tabela 3), algumas das variáveis que anteriormente se mostravam associadas com o desfecho perdem sua significância estatística e saem do modelo de análise, como a cor da pele, a renda per capita e o tabagismo. As variáveis idade e consumo de bebidas alcoólicas são mantidas no modelo para controlar as demais variáveis, mas também não se mostram associadas ao desfecho. Entre as variáveis socioeconômicas e demográficas, apenas a escolaridade e o sexo feminino permaneceram associados com o relato de DRGE, essa última mantendo a tendência de aumento do risco conforme a diminuição do grau de instrução dos indivíduos. O estado civil permanece associado, sendo maior o risco para indivíduos separados/divorciados.

As variáveis insônia, bem-estar psicológico e eventos estressantes mantêm-se fortemente associadas com o desfecho, mesmo quando se controla para possíveis fatores de confusão.

Entre as variáveis comportamentais, apenas o IMC mantémse associado com a ocorrência de sintomas de DRGE, com uma razão de chance de 1,68 para os mais obesos. 
TABELA 3 - Análise multivariada para sintomas de DRGE em Pelotas

\begin{tabular}{|c|c|c|c|}
\hline Característica & $\begin{array}{c}\text { Razão de } \\
\text { chance }\end{array}$ & $\begin{array}{c}\text { Intervalo de } \\
\text { confiança 95\% }\end{array}$ & $P$ valor \\
\hline Sexo & & & $<0,001$ \\
\hline Feminino & 1,39 & $1,20-1,61$ & \\
\hline Masculino & 1 & & \\
\hline Idade & & & 0,1 \\
\hline $20-29$ & 1,12 & $0,89-1,40$ & \\
\hline $30-39$ & 1,14 & $0,91-1,43$ & \\
\hline $40-49$ & 1,2 & $0,94-1,54$ & \\
\hline $50-59$ & 1,26 & $0,95-1,66$ & \\
\hline $60-69$ & 0,82 & $0,58-1,17$ & \\
\hline Mais de 69 & 1 & & \\
\hline \multicolumn{4}{|l|}{ Estado civil } \\
\hline Casado & 1 & & 0,02 \\
\hline Solteiro & 0,78 & $0,64-0,97$ & \\
\hline Viúvo & 0,92 & $068-1,23$ & \\
\hline Separado/divorciado & 1,25 & $0,97-1,61$ & \\
\hline Anos de escolaridade & & & $<0,001$ \\
\hline Não foi a escola & 3,91 & $2,86-5,35$ & \\
\hline 1 a 4 & 2,67 & $2,08-3,42$ & \\
\hline 5 a 8 & 2,22 & $1,76-2,80$ & \\
\hline 9 a 11 & 1,59 & $1,23-2,06$ & \\
\hline Mais de 11 & 1 & & \\
\hline Insônia & & & $<0,001$ \\
\hline Não & 1 & & \\
\hline Sim & 2,31 & $1,98-2,68$ & \\
\hline Bem-estar & & & $<0,001$ \\
\hline Alto & 1 & & \\
\hline Intermediário & 1,36 & $1,17-1,59$ & \\
\hline Baixo & 2,14 & $1,64-2,77$ & \\
\hline Eventos estressantes & & & 0,03 \\
\hline Nenhum & 1 & & \\
\hline Um & 1,01 & $0,86-1,19$ & \\
\hline Dois & 1,33 & $1,08-1,64$ & \\
\hline Três ou mais & 1,27 & $0,92-1,75$ & \\
\hline IMC & & & $<0,001$ \\
\hline$<20$ & 1 & & \\
\hline $2-24,99$ & 1,09 & $0,82-1,51$ & \\
\hline $25-29,99$ & 1,32 & $0,97-1,84$ & \\
\hline 30 ou mais & 1,68 & $1,21-2,4$ & \\
\hline Frequêencia de uso de álcool & & & 0,09 \\
\hline Não usa & 1 & $0,71-1,42$ & \\
\hline Menos de 3 vezes/semana & 0,87 & $0,61-1,24$ & \\
\hline $3-6$ vezes & 1,28 & $0,81-1,95$ & \\
\hline Diário & 1 & & \\
\hline
\end{tabular}

Quando se comparam as características da amostra obtida no presente estudo com os dados do censo realizado pelo IBGE em 2001, considerando-se apenas a população residente na zona urbana da cidade, é possível concluir que o processo amostral utilizado obteve bom resultado e que o estudo foi conduzido com amostra representativa da população da cidade, uma vez que as distribuições por sexo, idade, escolaridade e renda são semelhantes aos dados oficiais do IBGE para Pelotas.

O presente estudo utilizou para diagnóstico de DRGE os critérios mais comumente empregados em estudos anteriores. Comparando-se com a definição do atual Consenso Brasileiro sobre $\mathrm{DRGE}^{(9)}$, o critério utilizado foi mais sensível. A definição de sintomas presentes 2 vezes por semana durante, pelo menos, 4 a 8 semanas, utilizada pelo consenso, é mais específica. Sendo a DRGE uma enfermidade de evolução crônica, no entanto, é possível que o número de indivíduos sintomáticos 2 a 4 vezes por semana nas últimas 4 a 8 semanas não seja muito diferente dos que responderiam afirmativamente à presença de sintoma, pelo menos, uma vez por semana no último ano. Sendo diferente, a repercussão do uso de um critério mais sensível seria no sentido de superestimar a prevalência real. O efeito do critério diagnóstico utilizado sobre os reais fatores de risco para DRGE foi, provavelmente, conservador, uma vez que provoca diminuição da força de associação.

As dificuldades e limitações de delineamentos transversais em determinar fatores de risco para doenças são bem conhecidas ${ }^{(10)}$. Este estudo não está isento de viéses como o de causalidade reversa ${ }^{(12)}$, uma vez que não se pode determinar com precisão a cronologia da ocorrência de alguns fatores estudados e do surgimento dos sintomas de DRGE. O efeito de variáveis que representam hábitos de vida modificáveis pela presença de sintomas de DRGE, como tabagismo e consumo de bebidas alcoólicas, foi potencialmente alterado por este tipo de viés.

As variáveis socioeconômicas e demográficas estão, muito provavelmente, isentas deste tipo de erro, uma vez que se mantêm aproximadamente constantes ao longo da vida, não se alterando em conseqüência deste tipo de patologia. Mesmo a escolaridade já costuma estar bem definida na faixa etária estudada.

Para as variáveis insônia e bem-estar psicológico é possível a ocorrência deste viés, uma vez que os sintomas poderiam afetar a qualidade de vida e o sono dos pacientes. Sintomas digestivos, no entanto, não figuram entre as causas mais freqüentes de insônia relatada por pacientes ${ }^{(11)}$. Em geral, apenas pequena parte de indivíduos referiu alteração do seu ritmo de trabalho em decorrência de $\mathrm{DRGE}^{(8)}$ e a ocorrência de eventos estressantes não parece ser logicamente afetada pela patologia. Como as três variáveis tentam indicar fatores semelhantes e apresentam comportamento muito parecido no presente estudo, é razoável supor que este viés não tenha afetado de maneira importante os resultados destas variáveis.

As variáveis comportamentais, portanto, seriam as com maior potencial de alteração pela causalidade reversa. É possível que a presença de sintomas de DRGE tenha implicado em interrupção do hábito de fumar e de consumir bebidas alcoólicas, seja por recomendação médica, ou por auto-manejo dos indivíduos afetados.

A prevalência de sintomas de DRGE encontrada neste estudo é semelhante às encontradas em outros realizados em diferentes locais do mundo ${ }^{(1,2,10)}$, diferentemente da encontrada por KHOSHBATEN no Irã ${ }^{(6)}, 2,7 \%$ indicando provável diferença étnica na determinação dessa patologia. Os 31,3\% de prevalência encontrados no presente estudo encontram-se dentro da faixa de prevalências encontradas por $\mathrm{HEADING}^{(4)} \mathrm{em}$ seu estudo de revisão (21\% a 59\%), demonstrando, mais uma vez, a grande relevância desta patologia e sua importância para os serviços de saúde.

Há controvérsias na literatura quanto à associação entre sintomas de DRGE e o sexo. Alguns estudos encontraram maior risco para homens ${ }^{(1,5)}$, outros para mulheres ${ }^{(7)}$. Em todos os estudos revisados, os riscos relativos tanto para homens, quanto para mulheres são pequenos, nunca superiores a 1,5, o que pode sugerir que o sexo é pouco importante na determinação da ocorrência de sintomas de DRGE.

A ausência de associação entre DRGE e indivíduos nãobrancos é consistente com o achado de outros autores, uma vez que estudos em diferentes partes do mundo não têm encontrado relação com raça ou cor da pele $\mathrm{e}^{(1,5,10)}$.

O aumento do risco para desenvolvimento de sintomas de DRGE conforme diminui a faixa etária, encontrado neste estudo, é coerente 
com oa achados na Austrália ${ }^{(5)}$. A queda na prevalência entre aqueles com 70 ou mais anos de idade, pode ser explicada pelo menor número de indivíduos neste grupo, o que determina diminuição do poder de identificação da relação entre o desfecho e esta faixa etária.

Não parece haver plausibilidade biológica para justificar o efeito protetor de ser solteiro sobre a ocorrência de DRGE, como observado no atual estudo. Tal resultado deve-se, provavelmente, à associação dessa característica com outras não conhecidas e não medidas no mesmo.

A baixa escolaridade e a renda mais baixa que se mostram claramente associadas com a ocorrência de sintomas de DRGE na análise bruta, tem sua significância alterada, que se mantém apenas para a escolaridade após ajuste para fatores de confusão, fato compreensível pela alta colinearidade existente entre estas duas variáveis. A comparação destes achados com os de outros autores é dificultada por serem fatores pouco estudados, uma vez que a maioria das investigações sobre este tema foi conduzida em países desenvolvidos, onde as diferenças entre faixas de renda e de escolaridade não são marcantes.

Os achados de maior risco associados a todas as variáveis que envolvem aspectos psicológicos confirmam a grande importância destes fatores na determinação da doença. Outros estudos ${ }^{(1,2)}$ encontram resultados semelhantes. Ainda que as definições utilizadas para identificar problemas de origem psicológica sejam bastante diversas entre cada estudo, o aumento de risco aparece sempre que de alguma forma se estuda este tipo de exposição. Para garantir a comparabilidade e confirmar a importância desses achados, seria desejável que houvesse uma padronização dessa exposição em estudos futuros.
É nítida também a tendência de aumento de risco para sintomas de DRGE conforme aumenta o IMC dos indivíduos. No presente, somente a faixa de IMC correspondente à obesidade teve significância estatística, porém a faixa de sobrepeso apresentou um intervalo de confiança no limite da significância estatística, achado este em perfeito acordo com a literatura ${ }^{(2,10)}$.

A elevada prevalência de sintomas de DRGE detectada em Pelotas, associada ao grande desconforto causado ${ }^{(8,13)}$ e ao elevado número de consultas originadas por este tipo de sintoma ${ }^{(1)}$, bem como ao custo também elevado, ocasionado pela freqüente solicitação de exames complementares que estas consultas originam ${ }^{(1,13)}$, confirmam a necessidade de melhores estudos dos fatores determinantes da ocorrência da patologia. Programas de prevenção eficazes e protocolos clínicos adequados para a diminuição dos custos com a investigação da doença baseados no conhecimento da epidemiologia da DRGE no nosso meio, precisam ser desenvolvidos.

Atenção adequada aos indivíduos com relato de sintomas psicológicos e com sobrepeso/obesidade, particularmente tratando-se de mulheres com menos escolaridade, constitui-se em importante estratégia para a prevenção da DRGE.

Apesar da haver ampla publicação sobre este tema, a maioria dos estudos utiliza metodologia transversal e é baseada em serviços de saúde, o que superestima a prevalência real do desfecho na população. Uma vez que ainda persistem controvérsias sobre os fatores de risco para DRGE, seria recomendável investir em investigações com desenhos mais robustos, como os estudos de casos e controles e coortes.

Oliveira SS, Santos IS, Silva JFP, Machado EC. Gastroesophageal reflux disease: prevalence and associated factors. Arq Gastroenterol 42(2):116-121. ABSTRACT - Background - O gastroesophageal reflux is a high prevalent disease with prevalence rates ranging from 21 to $56 \%$ in different countries. Objective - To study the epidemiology and the prevalence of gastroesophageal reflux disease among adult population (20 years and old) at the city of Pelotas, southern Brazil. Methodology - A population-based cross-sectional study was conducted. Household interviews were done between October 1999 and January 2000. Association between gastroesophageal reflux disease and age, sex, skin color, years of formal education, income per capita, marital status, psychological variables (insomnia, stressful psychosocial events in the last year, psychological distress), body mass index, smoking and weekly consumption of alcoholic beverages was assessed through logistic regression. Results - Three thousands and nine-hundred thirty four 3.934 individuals were interviewed. A prevalence of $31,3 \%$ (CI $95 \%$ 29.9\%-32.8\%). After allowing for confounding gastroesophageal reflux disease was significantly associated with female sex, living without partner, low level of formal education, insomnia, psychological distress, reported stressful psychosocial events in the last year, obesity and overweight. Conclusion - Gastroesophageal reflux disease is a high prevalent disease and its main associated factors are sex, anthropometric variables and psychological characteristics.

HEADINGS - Gastroesophageal reflux, epidemiology. Prevalence. Risk factors.

\section{REFERÊNCIAS BIBLIOGRÁFICAS}

1. Bolin TD, Korman MG, Hansky J, Stanton R. Heartburn: community perceptions. J Gastroenterol Hepatol 2000;15:1-2

2. Diaz-Rubio M, Moreno-Elola-Olasco C, Rey E, Locke GR, Rodriguez-Artalejo F Symptoms of gastro-oesophageal reflux: prevalence, severity, duration and associated factors in Spanish population. Aliment Pharmacol Ther 2004;19:95-105.

3. Grainger SL, Klass HJ, Rake MO, Williams JG. Prevalence of dyspepsia: the epidemiology of overlapping symptoms. Postgrad Med J 1994;70:154-61.

4. Heading RC. Prevalence of upper gastrointestinal syptoms in the general population: a sistematic review. Scand J Gastroenterol 1999;231(suppl):3-8

5. Kay L, Jorgensen T, Jensen KH. Epidemiology of abdominal symptoms in a random population: prevalence, incidence, and natural history. Eur J Epidemiol 1994;10:559-66.

6. Khoshbaten M. Gatro-oesophageal reflux disease in northwestern Tabriz, Iran. Indian J Gastroenterol 2003;22:138-9.

7. Louis E, DeLooze D, Deprez P, Hiele M, Urbain D, Pelckmans P, Devière J, Deltenre M. Heartburn in Belgium: prevalence, impact on daily life, and utilization of medical resources. Eur J Gastroenterol Hepatol 2002;14(3):275-84.
8. McDowell I, Newell C. Measuring health. A guide to rating scales and questionnaires. New York: Oxford University Press; 1996

9. Moraes-Filho JPP, Gama-Rodrigues J, Meneghelli UG, Quigley E, Castro LP, Cecconello I. Brazilian Consensus on Gastroesophageal reflux disease: proposals for assessment, classification and management. Am J Gastoenterol 2002;97:241-8.

10. Nader F, Costa JSD, Nader GA, Motta GLCZ. Prevalência de pirose em Pelotas, RS, Brasil: estudo de base populacional. Arq Gastroenterol 2003;40:31-4.

11. Oliveira AV. Prevalência de insônia e fatores associados na população urbana adulta da cidade de Pelotas, RS [dissertação]. Pelotas: Universidade Federal de Pelotas; 2000

12. Rothman KJ, Greenland S. Modern epidemology. $2^{\text {nd }}$ ed. Philadelphia: Lippincott-Reave; 1998

13. Valle C, Broglia F, Pistonop A, Tinelli C, Perego M. Prevalence and impact of symptoms suggestive of gastroesophageal reflux disease. Dig Dis Sci 1999;44:1848-52.

14. Weihs C, Madisch A, Schlaud M, Heimann D, Mayer H, Hotz J. Socioepidemic analysis of sources of information and medical care for a subpopulation of female and male participants in a nationwide informative campaign in Germany on "Danger Signal: Heartburn". Gesundheitswesen 2003;65:402-8.

Recebido em 13/7/2004 Aprovado em 2/3/2005 